\title{
Beneficial Effect of Bendamustine in a Patient with Anti-MAG/SGPG Neuropathy and Bing-Neel Syndrome Associated with Waldenström Macroglobulinemia: A Case Report
}

\author{
Syugo Ueki ${ }^{a}$ Masataka Nakamura ${ }^{a}$ b Risa Sasaki $^{a} \quad$ Yoichiro Okada ${ }^{a}$ \\ Keisuke Yoshikawa ${ }^{d}$ Susumu Kusunoki ${ }^{d}$ Kazuyoshi Ishii $^{c}$ \\ Hirofumi Kusaka ${ }^{b}$ Takayuki Kondo ${ }^{a}$ \\ aDepartment of Neurology, Kansai Medical University Medical Center, Moriguchi, Japan; \\ ${ }^{b}$ Department of Neurology, Kansai Medical University, Hirakata, Japan; 'Department of \\ Internal Medicine, Kansai Medical University Medical Center, Moriguchi, Japan; \\ dDepartment of Neurology, Kindai University Faculty of Medicine, Osaka-Sayama, Japan
}

\section{Keywords}

Anti-MAG/SGPG neuropathy $\cdot$ Bing-Neel syndrome $\cdot \lg M$ paraprotein $\cdot$ Waldenström macroglobulinemia $\cdot$ Bendamustine

\begin{abstract}
A 71-year-old man with Waldenström macroglobulinemia (WM) presented with a slowly progressive sensory disturbance and mild weakness predominantly affecting the distal portion of the limbs over the course of 6 months. Cervical magnetic resonance imaging (MRI) showed a long hyperintense lesion at the C1-C4 level. Nerve conduction studies (NCS) revealed prolongation of distal latency, slowed conduction velocity, and conduction block. His serum IgM level was increased, and he was positive for anti-myelin-associated glycoprotein
\end{abstract}




\section{Case Reports in Neurology}

Case Rep Neurol 2018;10:88-94

DOI: $10.1159 / 000487850$

(C)

(C) 2018 The Author(s). Published by S. Karger AG, Basel www.karger.com/crn

Ueki et al.: Beneficial Effect of Bendamustine in a Patient with Anti-MAG/SGPG

Neuropathy and Bing-Neel Syndrome Associated with Waldenström Macroglobulinemia

(MAG) and anti-sulfoglucuronyl paragloboside (SGPG) IgM antibodies. Based on the presence of anti-MAG/SGPG antibodies and a single atypical cell with lymphoplasmacytic character in the cerebral spinal fluid, he was diagnosed as having anti-MAG/SGPG neuropathy and Bing-Neel syndrome (BNS) associated with WM. Following 6 cycles of bendamustine monotherapy, the patient's neurological impairment improved; and the serum IgM level became normalized. Furthermore, NCS findings indicated improvement; and the hyperintense lesion on MRI had almost completely disappeared. The present findings suggest that bendamustine monotherapy is effective not only for WM but also for its associated MAG/SGPG neuropathy and BNS.

(C) 2018 The Author(s)

Published by S. Karger AG, Basel

\section{Background}

Waldenström macroglobulinemia (WM) is a rare B-cell lymphoplasmacytic lymphoma characterized by the involvement of bone marrow, lymph nodes, and spleen, accompanied by the production of monoclonal immunoglobulin M (IgM) protein. Neurological complications develop in 50\% of patients with WM and typically consist of symmetrical sensorimotor peripheral neuropathy with IgM antibody against myelin-associated glycoprotein (MAG)/sulfated glucuronyl paragloboside (SGPG), but direct involvement of the central nervous system by neoplastic lymphoid cells can also occur [1], and this extremely rare phenomenon is referred to as Bing-Neel syndrome (BNS) [2]. Here, we report the case of a patient with anti-MAG/SGPG neuropathy and BNS associated with WM who was successfully treated with bendamustine monotherapy.

\section{Case Presentation}

A 71-year-old man with a 6-month history of slowly progressive gait disturbance as well as a tingling sensation and weakness in the distal end of all 4 limbs visited the Department of Orthopedic Surgery at Kansai Medical University. Cervical magnetic resonance imaging (MRI) showed spinal cord swelling and a long hyperintense lesion at the C1-C4 level (Fig. 1a, b); the lesion was partially enhanced by gadolinium (Fig. 1c, d). Given the possibility of myelitis, he was admitted to our department for further investigation. The patient's medical history revealed that he had been diagnosed with WM 9 years before the development of neurological complications, based on the presence of IgM- $\kappa$ type monoclonal protein and infiltration of lymphoplasmacytic cells identified in a bone marrow biopsy. Low-dose melphalan plus prednisolone therapy was initiated, and the serum IgM protein decreased. Six and a half years after the initial treatment with melphalan and prednisolone, the serum IgM protein increased again. So, he was treated with 4 cycles of rituximab, cyclophosphamide, and dexamethasone and then with 16 cycles of cyclophosphamide and dexamethasone because of infusion reactions due to the rituximab administration.

On admission, neurological examination revealed mild weakness of the distal muscles of bilateral upper limbs, an absence of the Achilles reflex, and symmetric distal impairment of 


\section{Case Reports in Neurology}

Case Rep Neurol 2018;10:88-94

DOI: $10.1159 / 000487850$

(c)

(C) 2018 The Author(s). Published by S. Karger AG, Basel www.karger.com/crn

Ueki et al.: Beneficial Effect of Bendamustine in a Patient with Anti-MAG/SGPG

Neuropathy and Bing-Neel Syndrome Associated with Waldenström Macroglobulinemia

pain and touch sensations in the upper limbs and of pain, touch, and vibration sensations in the lower limbs. Neither pyramidal sign nor sphincter disturbance was found.

Laboratory tests showed anemia with a hemoglobin level of $9.0 \mathrm{~g} / \mathrm{dL}$. Tests for autoantibodies including anti-nuclear, anti-SS-A, anti-SS-B, anti-double-stranded DNA, and antineutrophil cytoplasmic antibodies were all negative. Serum levels of IgM $(2,516 \mathrm{mg} / \mathrm{dL})$ and soluble interleukin-2 receptor $(8,730 \mathrm{U} / \mathrm{mL})$ were increased. A cerebrospinal fluid (CSF) analysis revealed an elevated protein level $(245 \mathrm{mg} / \mathrm{dL})$. CSF cytology identified a single atypical cell with lymphoplasmacytic character, prominent nucleoli, and basophilic cytoplasm with a perinuclear halo.

A brain MRI showed no abnormalities. Nerve conduction studies (NCS) revealed a pattern of demyelinating sensory-motor polyneuropathy (Table 1). Motor NCS examining the right median, ulnar, and tibial nerves revealed retardation of motor conduction velocities and prolongation of distal latency. A conduction block was detected in the right tibial nerve. Compound muscle action potential of the right peroneal nerve was not evoked. Sensory NCS in the right median and ulnar nerves showed low sensory nerve action potentials (SNAPs) with mildly reduced conduction velocities. SNAP of the right sural nerve was not evoked. Anti-MAG antibody was detected by western blot analysis.

Serum SGPG IgM antibody was detected by an enzyme-linked immunosorbent assay, whereas tests for serum IgM and IgG antibodies to GM1, GM2, GM3, GD1a, GD1b, GD3, GT1b, GQ1b, GA1, and Gal-C were all negative. Serum anti-AQP4, anti-MOG, and anti-neurofascin 155 antibodies were also negative. Therefore, we diagnosed the patient as having antiMAG/SGPG neuropathy and BNS associated with WM. The patient was given intravenous bendamustine (100-120 mg/m²), repeated every 4 weeks, for a total of 6 courses. Following treatment, his distal muscle power returned to normal, and his sensory disturbances improved dramatically. The serum IgM and sIL2-R levels declined to $167 \mathrm{mg} / \mathrm{dL}$ and 581 $\mathrm{U} / \mathrm{mL}$, respectively. Serum anti-SGPG antibody titer slightly reduced from 0.857 before treatment to 0.776 optical density at 400 dilution. Findings of a repeated NCS showed a partial improvement (Table 1). A follow-up MRI revealed nearly complete resolution of the previous abnormal cord signal changes and disappearance of the contrast enhancement (Fig. $1 \mathrm{e}-\mathrm{h})$.

\section{Discussion}

Our patient showed predominantly distal, slowly progressive, and predominantly sensory disturbance with relatively mild weakness. The results of the NCS fulfilled the electrophysiological criteria for demyelinating neuropathy. These clinical and electrophysiological findings and positive results for the anti-MAG/SGPG IgM antibodies were consistent with anti-MAG/SGPG neuropathy [3]. As additional specific electrophysiological features, antiMAG/SGPG neuropathy typically shows uniform symmetrical, and predominantly distal, reduced conduction velocity (terminal latency index $<0.25$ ) without conduction block [4], but all of the nerves studied in our patient had a terminal latency index of $>0.25$. Furthermore, our patient showed conduction block, which is commonly seen with chronic inflammatory demyelinating polyneuropathy but is not characteristic of anti-MAG/SGPG neuropathy. 


\section{Case Reports in Neurology}

Case Rep Neurol 2018;10:88-94

DOI: $10.1159 / 000487850$

(C)

(C) 2018 The Author(s). Published by S. Karger AG, Basel www.karger.com/crn

Ueki et al.: Beneficial Effect of Bendamustine in a Patient with Anti-MAG/SGPG

Neuropathy and Bing-Neel Syndrome Associated with Waldenström Macroglobulinemia

Based on the evidence regarding the pathogenicity of anti-MAG/SGPG antibodies, therapy is typically aimed at reducing the levels of circulating IgM or anti-MAG/SGPG antibodies by plasma exchange, intravenous immunoglobulin, corticosteroids, immunosuppressive agents, or interferon- $\alpha$ [4]. Recently, rituximab was shown to be beneficial for the treatment of anti-MAG/SGPG neuropathy [3]. However, there is inadequate reliable evidence to support any particular immunotherapy treatment for anti-MAG/SGPG neuropathy [5]. So far, only a single prior case report demonstrated the efficacy of bendamustine for the treatment of anti-MAG neuropathy, although bendamustine was used in combination with rituximab in this case [6]. Upon treatment with bendamustine, our patient showed improvement in sensory and motor symptoms and in NCS findings that correlated with a reduction in IgM levels. As shown in our present case, bendamustine monotherapy may also be effective for patients with anti-MAG/SGPG neuropathy who are not eligible for rituximab due to infusion-related side effects.

Our patient also presented with BNS. The clinical symptoms of BNS are diverse and mainly represented by unexplained limb weakness, altered mental status, and/or cranial nerve palsies [7]. Diagnostic considerations include brain and spinal MRI, CSF, and tissue biopsy. Despite the finding that his cervical MRI displayed a long hyperintense lesion with contrast enhancement, our patient did not have any clinical evidence of myelitis. Although this lesion was not confirmed histologically, our patient was diagnosed with BNS based on the abnormal MRI findings, CSF cytology, and history of WM. Treatment options for BNS include intrathecal and systemic chemotherapy, novel agents, and radiotherapy; but there is no standard treatment for patients with BNS. Recently, Minnema et al. [8] proposed an algorithm for the treatment of de novo BNS. Our patient was treated with bendamustine, which is recommended for symptomatic patients [8]. A follow-up MRI showed almost complete resolution of the abnormalities and no enhancement. Although the nature of the lesion remains unclear in the absence of biopsy, vasogenic edema following blood-brain barrier destruction caused by lymphoplasmacytic infiltration may present as abnormal signal intensities and contrast enhancement on MRI. MRI of the brain and spinal cord is recommended for patients with WM, even in the absence of central neurological symptoms. We should consider therapy for asymptomatic as well as symptomatic patients presenting with BNS.

\section{Conclusions}

We presented a patient with WM who developed immuno-mediated neuropathy with anti-MAG/SGPG antibodies and BNS. Bendamustine monotherapy appeared to have been effective for both anti-MAG/SGPG neuropathy and BNS. However, further studies are needed to determine the effectiveness of this chemotherapy.

\section{Acknowledgement}

We thank Dr. Toshiyuki Takahashi (Department of Neurology, Tohoku University Graduate School of Medicine) and Dr. Hidenori Ogata (Department of Neurology, Neurological 
Institute, Graduate School of Medical Sciences, Kyushu University) for measurement of the anti-MOG and anti-neurofascin 155 antibodies, respectively.

\section{Statement of Ethics}

As this case report does not meet the definition of human research, the authors declare that ethics approval was not required for this case report. We confirm that a signed consent from the patient has been obtained for the publication of this report.

\section{Disclosure Statement}

The authors declare that they have no competing interests.

\section{Funding Sources}

We received no funding support.

\section{References}

1 Baehring JM, Hochberg EP, Raje N, Ulrickson M, Hochberg FH: Neurological manifestations of Waldenström macroglobulinemia. Nat Clin Pract Neurol 2008;4:547-556.

2 Bing J, Neel A: Two cases of hyperglobulinemia with affection of the central nervous system on a toxiinfectious basis. Acta Med Scand 1936;88:492-506.

-3 Dalakas MC, Rakocevic G, Salajegheh M, Dambrosia JM, Hahn AF, Raju R, McElroy B: Placebo-controlled trial of rituximab in IgM anti-myelin-associated glycoprotein antibody demyelinating neuropathy. Ann Neurol 2009;65:286-293.

$\checkmark 4$ Joint Task Force of the EFNS and the PNS: European Federation of Neurological Societies/Peripheral Nerve Society Guideline on management of paraproteinemic demyelinating neuropathies. Report of a Joint Task Force of the European Federation of Neurological Societies and the Peripheral Nerve Society - first revision. J Peripher Nerv Syst 2010;15:185-195.

-5 Lunn MP, Nobile-Orazio E: Immunotherapy for IgM anti-myelin-associated glycoprotein paraproteinassociated peripheral neuropathies. Cochrane Database Syst Rev 2016;10:CD002827.

6 Gomez A, Hoffman JE: Anti myelin-associated-glycoprotein antibody peripheral neuropathy response to combination chemoimmunotherapy with bendamustine/rituximab in a patient with biclonal IgM $\kappa$ and $\operatorname{IgM} \lambda$ : case report and review of the literature. Clin Lymphoma Myeloma Leuk 2016;16:e101-e108.

7 Castillo JJ, D'Sa S, Lunn MP, Minnema MC, Tedeschi A, Lansigan F, Palomba ML, Varettoni M, Garcia-Sanz R, Nayak L, Lee EQ, Rinne ML, Norden AD, Ghobrial IM, Treon SP: Central nervous system involvement by Waldenström macroglobulinaemia (Bing-Neel syndrome): a multi-institutional retrospective study. Br J Haematol 2016;172:709-715.

8 Minnema MC, Kimby E, D’Sa S, Fornecker LM, Poulain S, Snijders TJ, Kastritis E, Kremer S, Fitsiori A, Simon L, Davi F, Lunn M, Castillo JJ, Patterson CJ, Le Garff-Tavernier M, Costopoulos M, Leblond V, Kersten MJ, Dimopoulos MA, Treon SP: Guideline for the diagnosis, treatment and response criteria for Bing-Neel syndrome. Haematologica 2017;102:43-51. 


\section{Case Reports in Neurology}
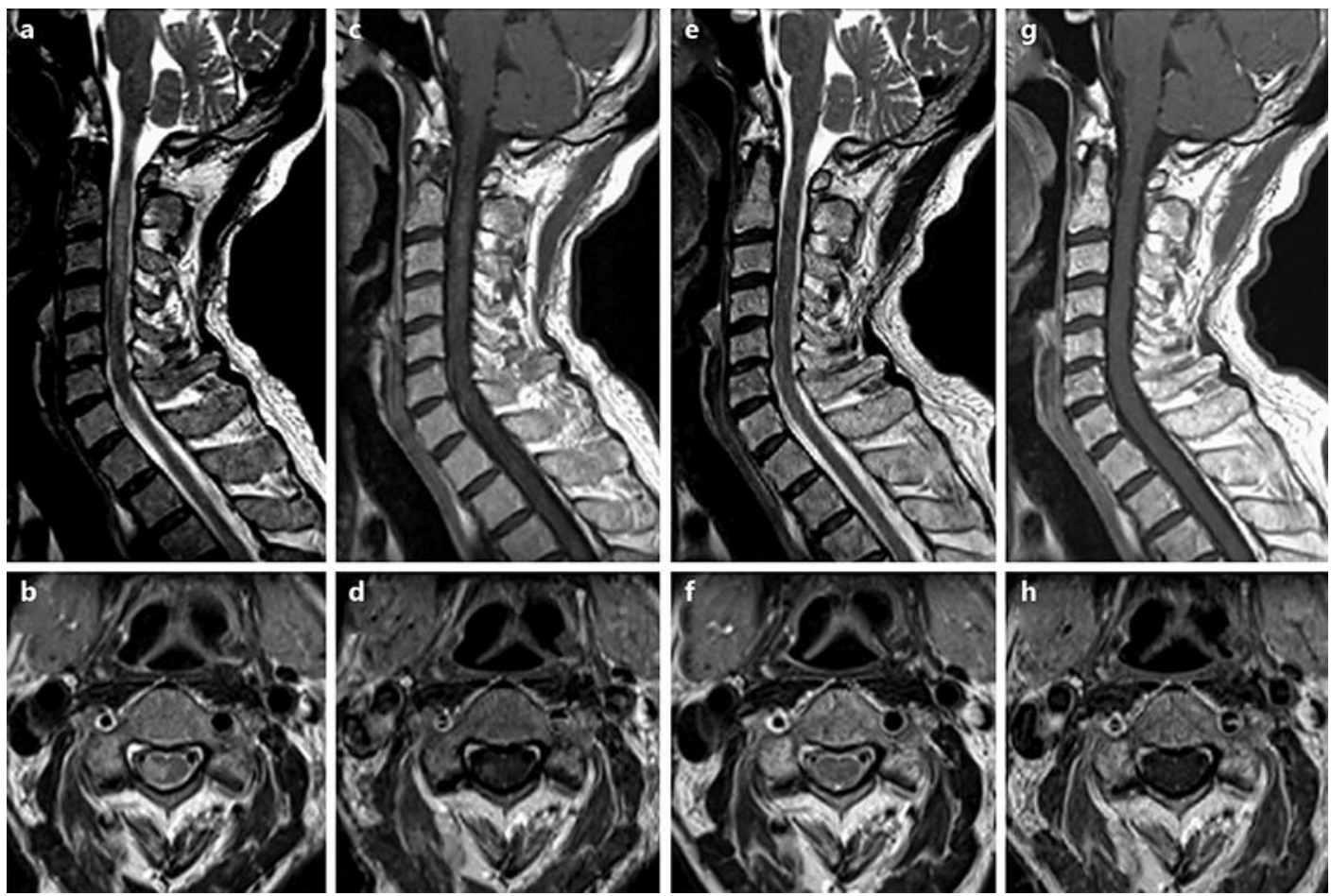

Fig. 1. a, b Sagittal and axial T2-weighted magnetic resonance (MR) images obtained on admission show a long hyperintense lesion at the C1-C4 level. c, $\mathbf{d}$ Sagittal and axial post-gadolinium T1-weighted MR images obtained on admission demonstrate focal enhancement. e-h Sagittal and axial T2-weighted MR images (e, f) and post-gadolinium T1-weighted MR images $(\mathbf{g}, \mathbf{h})$ obtained after the 6 cycles of treatment reveal near complete resolution of the previous lesion without enhancement. 
Table 1. Parameters of nerve conduction studies before and after therapy

\begin{tabular}{|c|c|c|c|c|c|c|c|c|c|}
\hline Nerve & $\begin{array}{l}\text { Bendamustine } \\
\text { therapy }\end{array}$ & $\begin{array}{l}\text { DL, } \\
\mathrm{ms}\end{array}$ & $\begin{array}{l}\text { CMAP, } \\
\mathrm{mV}\end{array}$ & $\begin{array}{l}\mathrm{MCV} \\
\mathrm{m} / \mathrm{s}\end{array}$ & $\begin{array}{l}\text { F latency, } \\
\text { ms }\end{array}$ & $\begin{array}{l}\text { SNAP, } \\
\mu \mathrm{V}\end{array}$ & $\begin{array}{l}\mathrm{SCV} \\
\mathrm{m} / \mathrm{s}\end{array}$ & TLI & $\mathrm{CB}$ \\
\hline \multirow[t]{2}{*}{ Median R } & before & 5.6 & 6.3 & 28.9 & 41.3 & 6.0 & 44.8 & 0.62 & - \\
\hline & after & 4.7 & 7.0 & 35.1 & 37.1 & 5.8 & 46.3 & 0.55 & - \\
\hline \multirow[t]{2}{*}{ Ulnar R } & before & 4.7 & 6.5 & 19.0 & 48.1 & 3.8 & 41.1 & 0.90 & - \\
\hline & after & 4.8 & 7.8 & 29.5 & 46.6 & 5.1 & 40.7 & 0.64 & - \\
\hline \multirow[t]{2}{*}{ Tibial R } & before & 6.4 & 1.1 & 39.1 & 63.2 & & & 0.40 & + \\
\hline & after & 6.8 & 3.8 & 29.8 & 65.3 & & & 0.44 & - \\
\hline \multirow[t]{2}{*}{ Peroneal R } & before & n.e. & n.e. & n.e. & n.e. & & & & n.e. \\
\hline & after & 6.3 & 0.5 & 36.0 & n.e. & & & 0.31 & - \\
\hline \multirow[t]{2}{*}{ Sural R } & before & & & & & n.e. & n.e. & & \\
\hline & after & & & & & n.e. & n.e. & & \\
\hline
\end{tabular}

$\mathrm{R}$, right; DL, distal latency; CMAP, compound muscle action potential; MCV, motor nerve conduction velocity; SNAP, sensory nerve action potential; SCV, sensory nerve conduction velocity; TLI, terminal latency index; $\mathrm{CB}$, conduction block; n.e., not evoked. 